\title{
Potential contribution of benzodiazepine abuse in the development of a bladder sarcomatoid carcinoma: A case report
}

\author{
RAFFAELE BAIO ${ }^{1}$, NICOLA SPIEZIA ${ }^{2}$, CARLA MARANI ${ }^{3}$ and MANLIO SCHETTINI ${ }^{2}$ \\ ${ }^{1}$ Department of Urology, University of Salerno, I-84081 Salerno; ${ }^{2}$ Department of Urology, Private Hospital 'Lourdes Clinic', \\ I-80040 Naples; ${ }^{3}$ Department of Anatomopathology, San Carlo Hospital of Nancy, I-00165 Rome, Italy
}

Received April 16, 2021; Accepted August 18, 2021

DOI: $10.3892 / \mathrm{mco} .2021 .2394$

\begin{abstract}
Invasive urothelial carcinoma has a propensity for disparate differentiation and presentation of morphological variants. Sarcomatoid carcinoma $(\mathrm{SaC})$ of the bladder is an extremely uncommon and aggressive variant of bladder cancer. An accurate diagnosis for this variant is necessary, but at times difficult. Immunohistochemistry can increase the diagnostic accuracy for $\mathrm{SaC}$. The therapeutic approaches currently adopted for the treatment of $\mathrm{SaC}$ are similar to those used for the urothelial variant: Surgery, chemotherapy and radiation therapy. To date, however, there exists no standard treatment due to the lack of knowledge regarding the pathogenesis of $\mathrm{SaC}$. Future research is required to focus on this rare histological tumor subtype in order to identify more effective treatment strategies. The present study reported an unusual case of bladder $\mathrm{SaC}$ in a woman aged 48 years, who was a non-smoker with a long history of benzodiazepine abuse. Although saving the patient's life was a priority, it was also essential to consider her subsequent quality of life. For that reason, the patient underwent a cystectomy with orthotopic neobladder reconstruction using ileum, followed by chemotherapy. At the 7 month follow-up, the patient was still alive, in complete remission and had normal bladder function. The present case report shows the potential contribution of benzodiazepine abuse in the development of a bladder sarcomatoid carcinoma, a rare variant of $\mathrm{BC}$, whose early detection and accurate diagnosis are key to attaining satisfactory treatment outcomes and a favorable prognosis for patients.
\end{abstract}

\section{Introduction}

Given that bladder cancer (BC) is the most frequent form of genitourinary tract cancer and taking into account the serious

Correspondence to: Dr Raffaele Baio, Department of Urology, University of Salerno, 43 Salvador Allende Street, Baronissi, I-84081 Salerno, Italy

E-mail: raffy.baio@yahoo.it

Key words: sarcomatoid carcinoma of the bladder, benzodiazepine abuse, radical cystectomy with pelvic lymphadenectomy nature of the presenting symptoms, treatment is urgent, due to the high recurrence and treatment-related morbidity associated with the disease (1). Smoking is the main risk factor for BC, accounting for 50-65\% of new cases in men and 20-30\% in women (2). Occupational exposure to formaldehydes, polycyclic aromatic hydrocarbons and other solvents is also considered to be a significant risk factor (3). A third identified risk factor is the application of a vesical catheter which, over a long period of time, can lead to chronic irritation of the vesical mucosa. Untreated vesical lithiasis and cystitis, as a result of Schistosoma hematobium infection, have been found to be causally linked to the onset of bladder squamous cell carcinoma. Urothelial carcinoma (UC), sometimes referred to as transitional cell carcinoma, is an occurring form of BC. However, UC has a propensity for disparate differentiation and presentation of morphological variants, including the sarcomatoid subtype. This variant has historically been given different names, such as sarcomatoid carcinoma $(\mathrm{SaC})$, pseudosarcoma, malignant mixed mesodermal/Müllerian tumor, metaplastic carcinoma and spindle cell carcinoma. Accounting for $\sim 0.3 \%$ of all primary urinary $\mathrm{BC}$ cases (1), $\mathrm{SaC}$ has similar epidemiological characteristics to those of UC, although they differ in terms of behavior and prognosis (4). As a rare malignant neoplasm of the urinary bladder, $\mathrm{SaC}$ is a high-grade biphasic neoplasm. Bladder $\mathrm{SaC}$ comprises two different components, one malignant epithelial component and one sarcomatoid component of mesenchymal origin. The latter component often manifests itself as a high-grade spindle cell neoplasm, while the former component may manifest itself as UC, squamous cell carcinoma, adenocarcinoma, small cell carcinoma or overlying carcinoma in situ. It frequently occurs in older patients, normally those in their sixth or seventh decade of life, and is three times more common in men than in women (5). A survey by the Mayo Clinic conducted between 1936 and 1995 showed that its frequency was higher in men aged 72 years, who had a history of smoking (6). The precise etiology of SaC has not yet been established, but radiation therapy and intravesical cyclophosphamide chemotherapy have been reported to enhance the risk of $\mathrm{SaC}$ and smoking has been identified as a major risk factor (5). In addition, the definite histogenesis of $\mathrm{SaC}$ is disputed. According to some studies, $\mathrm{SaC}$ is the result of the ability of undifferentiated, totipotential neoplastic cells to form multiple pathways of terminal differentiation in histologically recognized mesenchymal and epithelial elements. 
This view is based on the immunoreactivity of epithelial markers [cytokeratin and epithelial membrane antigen (EMA)] in mesenchymal areas. Other studies have suggested that $\mathrm{SaC}$ results from the 'collision' of two distinct malignant tumors that are mutually invasive. A third explanation is the possible occurrence of metaplasia of malignant epithelial elements into sarcomatoid elements. This theory is backed by the evidence of positive staining for keratins (AE1/AE3) in the epithelial and mesenchymal components $(7,8)$. The presentation of $\mathrm{SaC}$ is similar to that of urothelial carcinoma, with symptoms such as gross hematuria, dysuria, pollakiuria and urinary infection. Macroscopically, these tumors present as large exophytic polyps $(5-7 \mathrm{~cm}$ in diameter on average) with areas of necrosis and ulceration (7). However, the histopathological diagnosis of $\mathrm{SaC}$ can be challenging and it may be preferable for it to be combined with immunohistochemistry. Evidence in support of histological diagnosis includes H\&E and immunohistochemical staining results [positive staining for cytokeratins in the epithelial component and vimentin, desmin HHF-35, smooth muscle actin (SMA) or S100 calcium binding protein B in the mesenchymal component, respectively] $(7,9,10)$. This positive staining can differentiate this variant from other types of sarcoma (11). In effect, differential diagnosis of $\mathrm{SaC}$ with primary sarcomas, primary carcinomas with stromal metaplasia, carcinomas with pseudosarcomatous stroma, sarcomas associated with pseudoepitheliomatous hyperplasia, teratomas and prostate carcinomas with extension into the bladder is recommended. Considering that the incidence of divergent differentiation in cystectomy specimens is as high as $33 \%$, the fourth edition of the World Health Organization (WHO) classification of tumors of the urothelial tract provided a contemporary review of the morphology of urothelial neoplasms, including $\mathrm{SaC}$, within the group of invasive urothelial tumors (12). For this reason, according to the fourth edition of the 2016 WHO Classification of Tumors of the urinary Systema and Male Genital Organs, it is recommended that pathologists report the divergent histologies in their report (12). The clinical results of $\mathrm{SaC}$ are poorer than those of typical UC, usually including liver, lung and distant lymph node metastases and a postdiagnosis survival of 6 months. In effect, $\mathrm{SaC}$ is frequently diagnosed at an advanced local stage exhibiting nodal or distant metastasis. This rules out treatment approaches such as transurethral resection of bladder tumor or partial cystectomy; for all these reasons, the identification of this histological variant is clinically crucial and may require a multimodal approach (radical cystectomy plus adjuvant chemotherapy) for its treatment $(5,13)$. The present study reported a rare example of bladder $\mathrm{SaC}$ in a 48 -year-old woman without the commonly recognized risk factors of the variant.

\section{Case report}

A 48-year-old woman was admitted to our Urology Department after undergoing a transurethral bladder resection at another hospital. Histopathology of the resected Transurethral Resection of Bladder Tumor chips revealed a pT2 high-grade tumor. The physical examination and biochemical parameter investigation came back normal. The patient was not a smoker but had an almost 20-year history of benzodiazepine (BZD) abuse originally prescribed for treating anxiety and depression.
In particular, the patient was receiving alprazolam at a dose of $1 \mathrm{mg}$ twice daily in combination with a selective serotonin reuptake inhibitor (SSRI). However, she reported that, depending on the severity of her depression, she often exceeded the dosage recommended by the specialist. Furthermore, upon analyzing her clinical history, it emerged that the patient had undergone sleep treatment in adolescence. She had no family history of bladder neoplasms; furthermore, no additional urological and gynecological symptoms or diseases were identified. The computed tomography (CT) scan of the chest, abdomen and pelvis revealed that a huge mass was occupying almost the entire bladder without metastatic lesions. The mass exhibited enhancement upon the intravenous administration of contrast material. After signing written informed consent, the patient underwent radical cystectomy with bilateral extended pelvic lymphadenectomy, with neobladder reconstruction performed for urinary diversion. The most effective surgical technique for muscle-invasive $\mathrm{BC}$ is radical cystectomy which, in female patients, includes the removal of the uterus and parts of the ventral vagina, thus resulting in sterility (14). The patient underwent a total cystectomy with lymph node dissection, a total abdominal hysterectomy, as well as a bilateral salpingo-oophorectomy. During the surgery, ureters were exposed and split as close as possible to the urinary bladder; the urinary bladder was then resected and the uterus and both ovaries were removed. The urethra was carefully mobilized and exposed for subsequent neobladder anastomosis. Intraoperative pathology sections of the urethra and both ureters showed that they were tumor-free. Lymphatic tissue around the external iliac and internal iliac vessels and obturator nerve was dissected. The terminal ileum was identified and $45 \mathrm{~cm}$ of the ileum was isolated for the reconstruction of an orthotopic neobladder. The ureters were placed underneath the bowel in the orthotopic position. The neobladder remained intact, showing no leaks when filled with $180 \mathrm{ml}$ sodium chloride. A total of two ureteral stents and an indwelling catheter were placed intraoperatively. The follow-up was uneventful. The surgical specimen revealed an ulcerated polypoid mass with a $6,5 \mathrm{~cm}$ diameter, which involved almost the entire bladder; this mass presented a histopathological pattern of a high-grade muscle-invasive tumor with heterologous sarcomatoid elements, which consisted of elongated spindled cells (Fig. 1). Immunohistochemistry showed that these elements were positive for GATA binding protein 3, vimentin (Fig. 2), cytokeratin AE1/AE3 (Fig. 3) and EMA. The diagnosis reached was that of $\mathrm{SaC}$. In addition, the proliferation index, which was assessed with anti-Ki67 staining, was high $(60 \%)$, indicating the malignant nature of the lesion (Fig. 4). The TNM pathological stage was pT3a N2 M0 (G3, R0) (15). Therefore, adjuvant chemotherapy was deemed necessary. At the 6-month follow-up, the patient was alive and in complete remission, according to CT scans. Urodynamic testing and post-void residual urine investigation results showed that normal bladder function had been preserved. Table I summarizes the clinical and histopathological features of the patient and the treatment data.

\section{Discussion}

$\mathrm{UC}$ is one of the most common types of urinary $\mathrm{BC}$, accounting for $\sim 90 \%$ of tumors of this anatomic region. UC is known for 


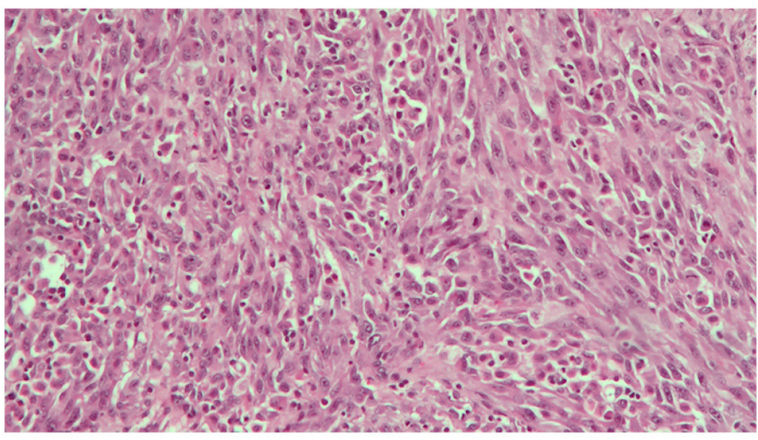

Figure 1. Presentation of UC with sarcomatoid changes (magnification, x10). Microscopically, the conventional urothelial component was mixed with the predominantly sarcomatous areas, which consisted of elongated spindled cells with vesicular, pleomorphic nuclei and conspicuous mitotic figures on a myxomatous background exhibiting a vaguely fascicular growth pattern. In this figure, obtained by hematoxolin-eosin staining, the nuclei appear colored as violet while the cytoplasm takes on a pink color.

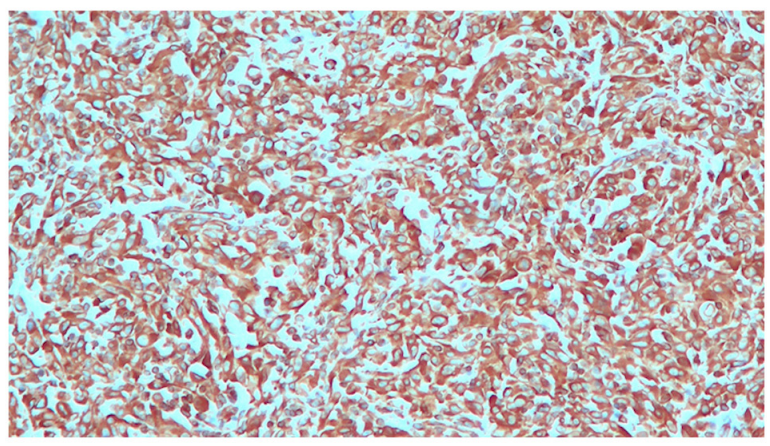

Figure 2. Tumor cells positive for vimentin expression. Vimentin is used as a marker of mesoderm-derived tissues and, for this reason, it is an immunohistochemical marker for sarcomas. This figure shows the positivity of bladder sarcomatoid cancer cells for vimentin expression (magnification, x10). Immunohistochemical staining for vimentin indicates cytoplasmatic positivity. The antigen-antibody immune reaction is revealed by a secondary antibody conjugated to a catalyst enzyme that reacts with a substrate, which forms a brown stain. While nuclei stain blue with hematoxylin.

its propensity towards disparate differentiation and presentation of morphological variants. The most frequent variant is the squamous variant, followed by the glandular variant (4). As bladder $\mathrm{SaC}$ is a rare and aggressive subtype of $\mathrm{BC}(16)$, there is a lack of data regarding its clinical outcomes and no effective treatments for the disease. Following a search of The Surveillance, Epidemiology and End Results database between 1988 and 2001, only 301 reported bladder SaC cases were identified out of 182,283 BC cases. This represented a mere $0.16 \%$ of all $\mathrm{BC}$ cases, highlighting the rarity of this condition. However, despite its rarity, this variant is a much more aggressive form of $\mathrm{BC}$.

The patient in the present study and her parents did not have a history of radiation therapy, cyclophosphamide exposure or smoking. However, the patient had an 20-year history of BZD abuse, originally prescribed for treating anxiety and depression. In particular, after having been prescribed alprazolam at a dosage of $1 \mathrm{mg}$ twice daily in combination with an SSRI by a specialist, the patient reported that she often exceeded the recommended dose, depending on the severity of her depression. BZDs have been prescribed on a

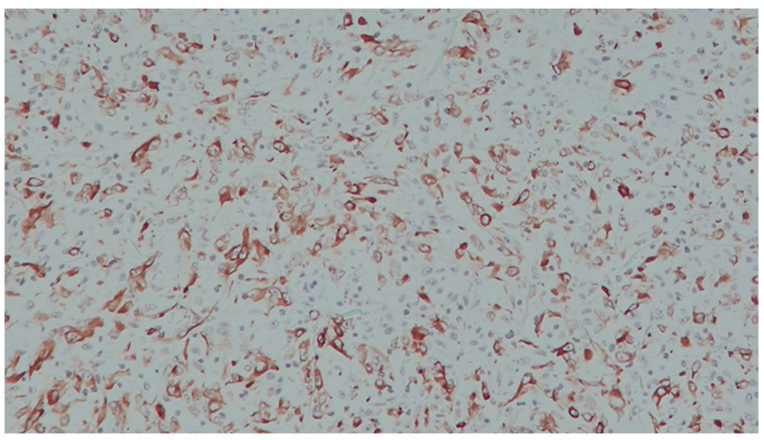

Figure 3. Focal positivity of bladder sarcomatoid cancer cells for high molecular weight cytokeratins. AE1/AE3 is a mixture of two monoclonal antibodies obtained by immunizing mice with human callus keratins, which identifies most human cytokeratins and can be used for positive identification of cells of epithelial origin on immunohistochemical examination (magnification, x10). Immunohistochemical staining for cytokeratins indicates cytoplasmatic positivity. The antigen-antibody immune reaction is revealed by a secondary antibody conjugated to a catalyst enzyme that reacts with a substrate, which forms a brown stain. Nuclei stain blue with hematoxylin.

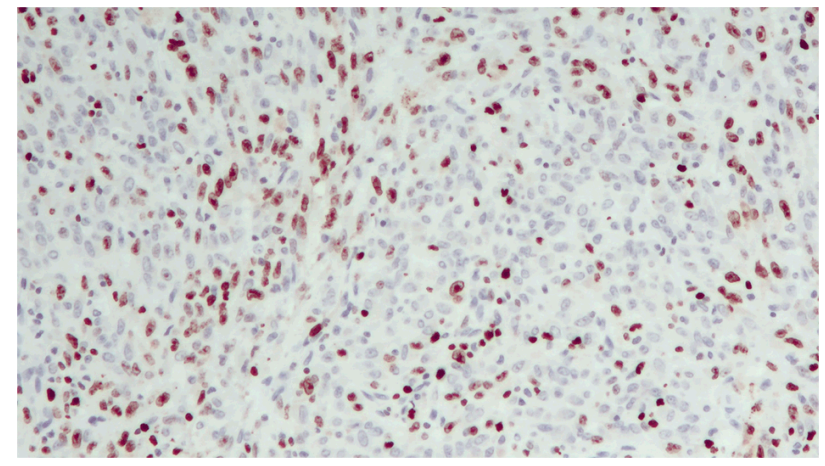

Figure 4. Immunohistochemical staining for Ki-67 expression, showing nuclear positivity, revealed a high proliferation index (magnification, x10). Nuclei of the replicating cells turn brown. Conversely, the nuclei of the cells that are not replicating are colored blue.

global scale for $\sim 50$ years, with a consumption rate of $10-42 \%$ in the elderly population. An association has been identified between the use of BZDs and a risk of cancer, but further research is required; to date, most studies have focused on cancer in animals exposed to BZDs $(17,18)$. Some of these studies have reported an association between carcinoma risks and the use of BZDs, more specifically between clonazepam and thyroid cancer (19), diazepam and breast cancer (18) and oxazepam and liver cancer (20). Iqbal et al (21) identified clonazepam, lorazepam, alprazolam, bromazepam, zolpidem and zopiclone as unsafe BZDs following exposure duration experiments. In particular, the study pointed to the high risk of cancer (15\%) associated with the use of clonazepam (21). However, the researchers admitted that this could possibly be an aggregated effect resulting from the combination of BZDs with long-term polypharmacy or metabolic drug use (22). The studies listed the increased risk for the development of various types of cancer as a result of BZDs exposure: Overall cancer risk increased by $21 \%$, brain cancer by $98 \%$, colorectal cancer by $25 \%$, lung cancer by $10 \%$, cancer of the esophagus by $59 \%$, prostate cancer by $36 \%$, BC by $39 \%$, liver cancer by $18 \%$, pancreatic cancer by $41 \%$ and other types of cancer 
Table I. Clinical and histopathological features and treatment data.

Clinicopathological features

Patient

Age, years

Sex

Usual risk factor (smoking, occupational exposure to solvents and bladder chronic irritation)

Past history

Tumour-stage and grade after endoscopic bladder resection

Tumour-stage and grade after radical treatment

(radical cystectomy with lymph node dissection)

State of surgical margins (negative or positive)

Urinary diversion type

Adjuvant treatment (yes or not)

Bladder cancer subtype

Proliferation index (anti-Ki67)
48

Female

None

Benzodiazepine abuse (for 20 years)

T2G3

T3aN2M0 (G3)

Negative (R0)

Orthotopic neobladder

Yes (adjuvant chemotherapy)

Sarcomatoid bladder cancer (positivity for GATA3, Vimentin, Cytokeratin AE1/AE2 and epithelial membrane antigen.

$60 \%$ by $27 \%$ (21). These findings have important implications for BZDs users, particularly considering the frequency of their prescriptions. The findings of Iqbal et al were supported by the findings of other studies, such as those of Rosenberg et al (23), Kripke and Langer (24) and Cronin-Fenton et al (25). BC is the sixth most common type of cancer worldwide, with its incidence rates being considerably high in Taiwan, where BZD usage has been associated with the risk of cancer (21). Studies by Kao et al (26) and Coogan et al (27) found that BZD use in males was particularly linked to a risk of prostate cancer.

Despite the rarity of this variant and the difficulty in reaching a diagnosis, $\mathrm{SaC}$ merits specific investigations, due to its aggressive nature and the poor prognosis associated with the disease (28). The best predictor of survival for $\mathrm{SaC}$ is the pathological stage at which it is diagnosed (28). Favorable prognostic characteristics include negative surgical margins and metastatic disease absence at first presentation. At the 2-year stage, the mortality rate is as high as $70 \%$. In the majority of patients, mortality is often caused by local disease or by lymph node, lung, pleura, brain, liver or bone metastasis. Survival prospects are even poorer for sarcomatoid variants than for organ-confined and metastatic UC. Due to the relative rarity of the disease and, consequently, the absence of findings from randomized controlled trials, the experts' opinions with regards to the most effective treatment are divided. Evidence suggests that the overall survival rate is higher following radical cystectomy with pelvic lymphadenectomy (14,29,30). For this reason, it is considered the principal approach; however, patients may develop local recurrence following surgery (16). In an attempt to prevent recurrence and distance metastasis, certain adjuvant treatment approaches, such as radiotherapy or chemotherapy, are often recommended.

In conclusion, $\mathrm{SaC}$ is a rare variant of $\mathrm{BC}$, whose early detection and accurate diagnosis are key to attaining satisfactory treatment outcomes and a favorable prognosis for patients. Histopathology and immunohistochemistry are crucial to achieving a timely diagnosis and management of this condition. The present study reported a case of bladder
$\mathrm{SaC}$ in a 48-year-old patient, thereby raising awareness for the incidence of this malignancy in a younger population than usually recorded. The present case was noteworthy due to the age of the patient and the total absence of the usual risk factors for $\mathrm{SaC}$. With regards to the causal factors of this disease, several studies cited herein have identified a large number of chemical compounds believed to be carcinogenic. The majority of them are aromatic amines and benzene derivatives; for that reason, there is a very high likelihood that long-term BZD abuse played a fundamental role in tumor development in the reported case.

\section{Acknowledgements}

Not applicable.

\section{Funding}

No funding was received.

\section{Availability of data and materials}

All data generated or analyzed during this study are included in this published article.

\section{Authors' contributions}

RB and NS confirm the authenticity of all the raw data. RB made substantial contributions to conception and design of this case report and to acquisition of data and was the major contributor to the writing of the manuscript. MS, NS and $\mathrm{CM}$ analyzed and interpreted the patient data regarding the urological disease. All authors have read and approved the final manuscript.

\section{Ethics approval and consent to participate}

Not applicable. 


\section{Patient consent for publication}

Signed informed consent was obtained from the patient for publication of this case report and any accompanying images.

\section{Competing interests}

The authors declare that they have no competing interests.

\section{References}

1. Lucon AM and Falci R Jr: Câncer de Bexiga. Vol II.Lopes AC (ed). Tratado de ClínicaMédica, São Paulo, pp2923-2930, 2006.

2. Freedman ND, Silverman DT, Hollenbeck AR, Schatzkin A and Abnet CC: Association between smoking and risk of bladder cancer among men and women. JAMA 306: 737-745, 2011.

3. Witjes JA, Compérat E, Cowan NC, De Santis M, Gakis G, Lebret T, Ribal MJ, Van der Heijden AG and Sherif A: EAU guidelines on muscle-invasive and metastatic bladder cancer: Summary of the 2013 guidelines. Eur Urol 65: 778-792, 2014.

4. Eble JN, Sauter G Sesterhenn I and Epstein JI (eds): Pathology and Genetics of Tumors of the Urinary System and Male Genital Organs. IARC Press, Lyon, pp99-133, 2004.

5. Wang J, Gillaspie C, Kunadharaju R, Talmon GA and Enke C: Sarcomatoid urothelial carcinoma: A single cancer center experience. World J Oncol 2: 175-180, 2011.

6. DeSantis CE, Lin CC, Mariotto AB, Siegel RL, Stein KD, Kramer JL, Alteri R, Robbins AS and Jemal A: Cancer treatment and survivorship statistics, 2014. CA Cancer J Clin 64: 252-271, 2014.

7. Silva CB, Alves MC, Ribeiro JC, Garcia P and Santos AR: Carcinoma Sarcomatóide da Bexiga. Acta Urológica 23: 61-64, 2006.

8. Venyo AK and Titi S: Sarcomatoid variant of urothelial carcinoma (carcinosarcoma, spindle cell carcinoma): A review of the literature. ISRN Urol 2014: 794563, 2014.

9. Babjuk M, Burger M, Zigeuner R, Shariat SF, van Rhijn BW, Compérat E, Sylvester RJ, Kaasinen E, Böhle A, Palou Redorta J and Rouprêt M: EAU guidelines on non-muscle-invasive urothelial carcinoma of the bladder: Update 2013. Eur Urol 64: 639-653, 2013.

10. Ploeg M, Aben KK and Kiemeney LA: The present and future burden of urinary bladder cancer in the world. World J Urol 27 289-293, 2009.

11. Sung MT, Wang M, MacLennan GT, Eble JN, Tan PH, Lopez-Beltran A, Montironi R, Harris JJ, Kuhar M and Cheng L: Histogenesis of sarcomatoid urothelial carcinoma of the urinary bladder: Evidence for a common clonal origin with divergent differentiation. J Pathol 211: 420-430, 2007.

12. Humphrey PA, Moch H, Cubilla AL, Ulbright TM and Reuter VE: The 2016 WHO classification of tumours of the urinary system and male genital organs-part B: Prostate and bladder tumours. Eur Urol 70: 106-119, 2016.

13. Mallik AU, Rahman MZ and Sarker MMR: Sarcomatoid carcinoma of urinary bladder-a case report. Banglajol 4: 28-29, 2010.

14. Kaufman DS, Shipley WU and Feldman AS: Bladder cancer. Lancet 374: 239-249, 2009.

15. Sobin LH, Gospodarowicz MK and Wittekind C: TNM Classification of Malignant Tumours. 7th edition. Wiley-Liss, New York, NY, 2010.
16. Wright JL, Black PC, Brown GA, Porter MP, Kamat AM, Dinney CP and Lin DW: Differences in survival among patients with sarcomatoid carcinoma, carcinosarcoma and urothelial carcinoma of the bladder. J Urol 178: 2302-2306, 2007.

17. Stopper H, Körber C, Spencer DL, Kirchner S, Caspary WJ and Schiffmann D: An investigation of micronucleus and mutation induction by oxazepam in mammalian cells. Mutagenesis 8: 449-455, 1993

18. Karmali RA, Volkman A, Muse P and Louis TM: The influence of diazepam administration in rats bearing the R3230AC mammary carcinoma. Prostaglandins Med 3: 193-198, 1979.

19. Miyawaki I, Moriyasu M, Funabashi H, Yasuba M and Matsuoka N: Mechanism of clobazam-induced thyroidal oncogenesis in male rats. Toxicol Lett 145: 291-301, 2003.

20. Iida M, Anna CH, Hartis J, Bruno M, Wetmore B, Dubin JR, Sieber S, Bennett L, Cunningham ML, Paules RS, et al: Changes in global gene and protein expression during early mouse liver carcinogenesis induced by non-genotoxic model carcinogens oxazepam and Wyeth-14,643. Carcinogenesis 24: 757-770, 2003.

21. Iqbal U, Nguyen PA, Syed-Abdul S, Yang HC, Huang CW, Jian WS, Hsu MH, Yen Y and Li YJ: Is long-term use of benzodiazepine a risk for cancer? Medicine (Baltimore) 94: e483, 2015.

22. Xu W, Tamim H, Shapiro S, Stang MR and Collet JP: Use of antidepressants and risk of colorectal cancer: A nested case-control study. Lancet Oncol 7: 301-308, 2006.

23. Rosenberg L, Palmer JR, Zauber AG, Warshauer ME, Strom BL, Harlap S and Shapiro S: Relation of benzodiazepine use to the risk of selected cancers: Breast, large bowel, malignant melanoma, lung, endometrium, ovary, non-Hodgkin's lymphoma, testis, Hodgkin's disease, thyroid, and liver. Am J Epidemiol 141: 1153-1160, 1995.

24. Kripke DF and Langer RD: Evidence for harm, comment on 'Use of benzodiazepines or benzodiazepine related drugs and the risk of cancer: A population-based case-control study'. Br J Clin Pharmacol 78: 186-187, 2013

25. Cronin-Fenton DP, Riis AH, Lash TL, Dalton SO, Friis S, Robertson D and Sørensen HT: Antidepressant use and colorectal cancer risk: A Danish population-based case-control study. $\mathrm{Br}$ J Cancer 104: 188-192, 2011.

26. Kao CH, Sun LM, Su KP, Chang SN, Sung FC, Muo CH and Liang JA: Benzodiazepine use possibly increases cancer risk: A population-based retrospective cohort study in Taiwan. J Clin Psychiatry 73: e555-e560, 2012.

27. Coogan-PF, Rosenberg L, Palmer JR, Strom BL, Stolley PD, Zauber AG and Shapiro S: Risk of ovarian cancer according to use of antidepressants, phenothiazines, and benzodiazepines (United States). Cancer Causes Control 11: 839-845, 2000.

28. Lopez-Beltran A, Pacelli A, Rothenberg HJ, Wollan PC, Zincke H, Blute ML and Bostwick DG: Carcinosarcoma and sarcomatoid carcinoma of the bladder: Clinicopathological study of 41 cases. J Urol 159: 1497-1503, 1998.

29. Bansal A, Kumar N and Sharma SC: Sarcomatoid variant of urothelial carcinoma of the urinary bladder. J Cancer Res Ther 9: 571-573, 2013.

30. Mittal V, Rupala KK, Yadav R and Suryavanshi M: Giant sarcomatoid carcinoma with osseous metaplasia from urinary bladder diverticulum. Indian J Surg Oncol 8: 436-439, 2017.

This work is licensed under a Creative Commons Attribution-NonCommercial-NoDerivatives 4.0 International (CC BY-NC-ND 4.0) License. 